\title{
Citron-Kinase, a Protein Essential to Cytokinesis in Neuronal Progenitors, Is Deleted in the Flathead Mutant Rat
}

\author{
Matthew R. Sarkisian, ${ }^{1}$ Weiwei Li, ${ }^{1}$ Ferdinando Di Cunto, ${ }^{2}$ Santosh R. D’Mello, ${ }^{3}$ and Joseph J. LoTurco ${ }^{1}$ \\ ${ }^{1}$ Department of Physiology and Neurobiology, University of Connecticut, Storrs, Connecticut 06269, ${ }^{2}$ Department of \\ Genetics, Biology and Biochemistry, University of Torino, 10126 Torino, Italy, and ${ }^{3}$ Department of Molecular and Cell \\ Biology, University of Texas at Dallas, Richardson, Texas 75083
}

\begin{abstract}
Cytokinesis is an essential step in neurogenesis, yet the mechanisms that control cytokinesis in the developing CNS are not well understood. The flathead $(f h)$ mutation in rat results in cytokinesis failure in neural progenitors followed by apoptosis and a dramatic reduction in CNS growth. Here we present evidence that the fh mutation is caused by a single base deletion in exon 1 of the gene encoding Citron-Kinase (Citron$\mathrm{K})$. This base deletion causes a premature stop codon at the 27th codon in the $\mathrm{N}$-terminal kinase domain of Citron-K, and Western blot and immunocytochemical analysis show that the Citron-K protein is absent in proliferative zones in fh/fh mutant embryos. We find that Citron-K protein is normally expressed
\end{abstract}

The specific proteins that regulate the pattern and progression of cytokinesis in the developing CNS are currently poorly defined. Analysis of spontaneous mutations in rodents and humans has led to the identification of proteins essential to many aspects of neural development, most notably migration (Feng and Walsh, 2001). The Flathead $(f h)$ mutation in rat is a relatively recent spontaneous, autosomal recessive mutation located on the long arm of rat chromosome 12 (Cogswell et al., 1998). The cellular phenotype of $f h / f h$ mutants includes abnormally high levels of cell death within and just outside of proliferative zones (Roberts et al., 2000; Sarkisian et al., 2001) and a failure in cytokinesis (Mitchell et al., 2001; Sarkisian et al., 2001). Recent analyses of disrupted neurogenesis in the flathead mutant indicate that the failure in cytokinesis precedes the increase in apoptosis (Mitchell et al., 2001; Sarkisian et al., 2001), suggesting that the primary molecular defect in the flathead mutant acts by disrupting cytokinesis in neuronal progenitors.

Genetic approaches, primarily in yeast, have led to the identification of many proteins that play a role in regulating cytokinesis (Chang and Nurse, 1996; Field et al., 1999). In general, the network of identified molecules interacts with cytoskeletal ele-

Received Oct. 17, 2001; revised Jan. 14, 2002; accepted Jan. 28, 2002.

This research was supported by National Institutes of Health Grant MH56524 and March of Dimes Grant 0307 to J.J.L. We thank Dr. Akiko Nishiyama and Jie Bu for help with Western blots, Dr. Marie Cantino and Steve Daniels for their help with the electron microscopy, and Dr. Joe Crivello and James Goldmeyer for help with the DNA sequencing portion of this study. We also thank Dr. Shuh Narumiya for providing us with a polyclonal rabbit anti-Citron antibody. The Rat401(Nestin) antibody developed by S. Hockfield was obtained from the Developmental Studies Hybridoma Bank developed under the auspices of the National Institute of Child Health and Human Development and maintained by The University of Iowa, Department of Biological Sciences (Iowa City, IA).

Correspondence should be addressed to Dr. Joseph J. LoTurco, Department of Physiology and Neurobiology, University of Connecticut, U-156, 3107 Horsebarn Hill Road, Storrs, CT 06269. E-mail: loturco@oracle.pnb.uconn.edu.

Copyright (ㄷ) 2002 Society for Neuroscience $0270-6474 / 02 / 220001-05 \$ 15.00 / 0$ along the ventricular zone (VZ) surface and localizes to cleavage furrows of both symmetrically and asymmetrically dividing progenitors. In addition, Citron-K colocalizes with RhoA at cleavage furrows in wild-type $(w t)$ embryos, whereas RhoA expression is reduced at the VZ surface and is absent from many cytokinesis furrows in homozygous fh/fh mutants. These results, together with evidence from a recently described induced mutation in mice, indicate that the flathead mutation is in the Citron-K gene and that Citron-K may act with RhoA to ensure the progression of cytokinesis in neuronal progenitors.

Key words: Citron; cortical malformation; epilepsy; progenitor; mitosis; neurogenesis; neocortex; Rho ments, including actin and tubulin, to ensure the appropriate location and constriction of the cleavage furrow (Drees et al., 2001). Recently, the small GTPase Rho has been implicated in playing a critical role during the contractility process in cytokinesis (Madaule et al., 1998, 2000). Similarly, Citron-Kinase (Citron-K), a Rho target the kinase activity of which is increased eightfold by activated Rho (Di Cunto et al., 1998), locates to the midbodies of dividing cells and appears to be critical for the normal contractility of the cleavage furrow (Madaule et al., 1998, 2000).

In a previous study, we mapped the $f h$ mutation to within a 1 cm interval on rat chromosome 12 (Cogswell et al., 1998). The homologous region of the human genome contains the gene encoding Citron-K, and therefore we hypothesized that a mutation in Citron-K may be the $f h$ mutation. Furthermore, a recent mouse knock-out of Citron-K (Di Cunto et al., 2000) shows disruptions in cytokinesis similar to those seen in the $f h / f h$ mutant rat. Here we report that $f h / f h$ rats have a single base deletion within exon 1 of the kinase domain of the Citron-K gene and that Citron-K protein is missing in $f h / f h$ mutants. Citron-K protein is highly polarized within neuroepithelium and localizes

This article is published in The Journal of Neuroscience, Rapid Communications Section, which publishes brief, peerreviewed papers online, not in print. Rapid Communications are posted online approximately one month earlier than they would appear if printed. They are listed in the Table of Contents of the next open issue of JNeurosci. Cite this article as: JNeurosci, 2002, 22:RC217 (1-5). The publication date is the date of posting online at www.jneurosci.org.

http://www.jneurosci.org/cgi/content/full/6283 
to the surface of the ventricular zone (VZ) and to cleavage furrows of both symmetrically and asymmetrically dividing cells in developing neocortex. Finally, we show that Citron-K colocalizes with its activator RhoA at cytokinesis furrows and that the normal expression pattern of RhoA at the $\mathrm{VZ}$ surface is disrupted in $f h / f h$ mutants. Together, these findings show that Citron-K is an essential regulator of cytokinesis in the developing CNS.

\section{MATERIALS AND METHODS}

Identification of the mutation in Citron Kinase. Five pairs of primers were used to clone the entire Citron-K coding sequence from cDNA prepared from total RNA isolated from embryonic day (E) $15 w t$ and $f h / f h$ telencephalons (SuperScript Preamplification System for First Strand cDNA Synthesis, Invitrogen, Gaithersburg, MD). Primers $\left(5^{\prime}-3^{\prime}\right)$ were as follows: kinase domain: GAGTCGGTAGCGGAGAGATGTT and CCCGACACAACAGACTCAGATC; Citron-nonkinase $(\mathrm{N})$ domain: GTGTGCTAGAGAAGTGACTGCG and CCTCATCGAGTTGTTTGGACAG, TCGCAACAGCTGTACTGTCATC and CATCTGCTTTGGCTGTATTTGC, TATCTATTCATGGTGCCGTTG and AGGAGGAGTTCTTCAGGCTGAG. PCR products were cloned into TOPO vector using the TA cloning kit (Invitrogen, Carlsbad, CA), and inserts were sequenced in both directions on a Beckman Coulter CEQ 2000 sequencer using a CEQ Dye Terminator Cycle Sequencing Kit (Beckman Coulter, Fullerton, CA). To rule out the possibility of introduced sequence errors from PCR, at least two separate clones from at least two different PCR reactions were sequenced. Furthermore, exon 1 of the Citron-K gene from genomic DNA of mutants and wt was amplified, cloned, and sequenced as above. The primers to exon 1 of the Citron-K gene in rat were based on sequence of mouse exon 1 and were GAGATGTTGAAGTTCAAGTA and CCTGGAAGAAGAGATTTAGC.

A P1 contig was constructed that contained the genetic interval that spanned the flathead mutation. P1 clones were isolated by PCR from a gridded genomic library (ratPAC1, Genome Systems, Cambridge, UK) with two simple sequence length polymorphism (sslp) primers, D12Rat80 and D12 Rat55 (Research Genetics, Huntsville, AL). These two sslp markers were determined in a genetic mapping study involving $181 \mathrm{~F} 2$ mutants (362 meioses) to be within $1 \mathrm{~cm}$ and flanking the flathead mutation. One P1 clone was isolated with D12Rat80, and three were isolated with D12Rat55 (Research Genetics). Each end of these P1 clones was sequenced, and PCR was used to determine the alignment of the four P1s in a contig. Primers to exon 1 of the Citron-K gene (above) were used to identify the location of the Citron gene within the contig. Each of the three P1s isolated with the D12Rat55 sslp marker contained sequence for the Citron-K gene.

Western blotting. Protein extracts from cerebral cortex and cerebellum were collected from either E13 or P1 $w t$ and $f h / f h$ rats. Tissues were homogenized in $2 \times$ SDS sample buffer $(6 \%$ SDS, $40 \%$ glycerol, $125 \mathrm{~mm}$ Tris, $\mathrm{pH} 6.8,10 \% \beta$-mercaptoethanol) and incubated for $10 \mathrm{~min}$ at $95^{\circ} \mathrm{C}$. Equivalent amounts of total protein were run on a $4-12 \%$ Tris-Glycine gel (Novex, San Diego, CA) and transferred onto polyvinylidene difluoride membranes (Millipore, Bedford, MA) at $350 \mathrm{~mA}$ for $1.5 \mathrm{hr}$. Membranes were blocked overnight at $4{ }^{\circ} \mathrm{C}$ in $5 \%$ nonfat dry milk (NFDM) in Tris-buffered saline containing $0.1 \%$ Tween (TBST). Immunoblotting was performed with the following primary antibodies: polyclonal rabbit anti-Citron primary antibody (1:3000) (Di Cunto et al., 2000), a monoclonal mouse anti-CRIK (1:500) (BD PharMingen, San Diego, CA), and polyclonal rabbit anti- $\beta$ catenin (1:4000) (Sigma, St. Louis, MO) in $2.5 \%$ NFDM in TBST for $3 \mathrm{hr}$ at room temperature. The membrane was washed several times with TBST and incubated for $1 \mathrm{hr}$ at room temperature with either a horseradish peroxidase goat antirabbit or goat anti-mouse secondary antibody (Bio-Rad, Hercules, CA) diluted 1:10,000 in 2.5\% NFDM in TBST. Membranes were developed using an ECL detection kit (Amersham Biosciences, Piscataway, NJ).

Immunocytochemistry. Forebrains from $w t$ and $f h / f h$ rats were examined at the following ages: E13, E14, and P3. All embryonic brains were collected into cold HBSS (Invitrogen) and fixed with $4 \%$ paraformaldehyde (PF) in $0.1 \mathrm{M}$ phosphate buffer, whereas postnatal brains were perfused with PBS followed by $4 \%$ PF. Brains were sectioned at 10-14 $\mu \mathrm{m}$ on a cryostat in either the coronal or sagittal planes. Sections for Citron and RhoA staining were pretreated in pepsin $(0.1 \mathrm{mg} / \mathrm{ml})$ in $0.1 \mathrm{~N}$ $\mathrm{HCl}$ for $30 \mathrm{sec}-3 \mathrm{~min}$ followed by standard immunostaining procedures. Primary antibodies used on sections or fixed cells were as follows: polyclonal rabbit anti-Citron(1:500) (a gift from S. Narumiya, Kyoto
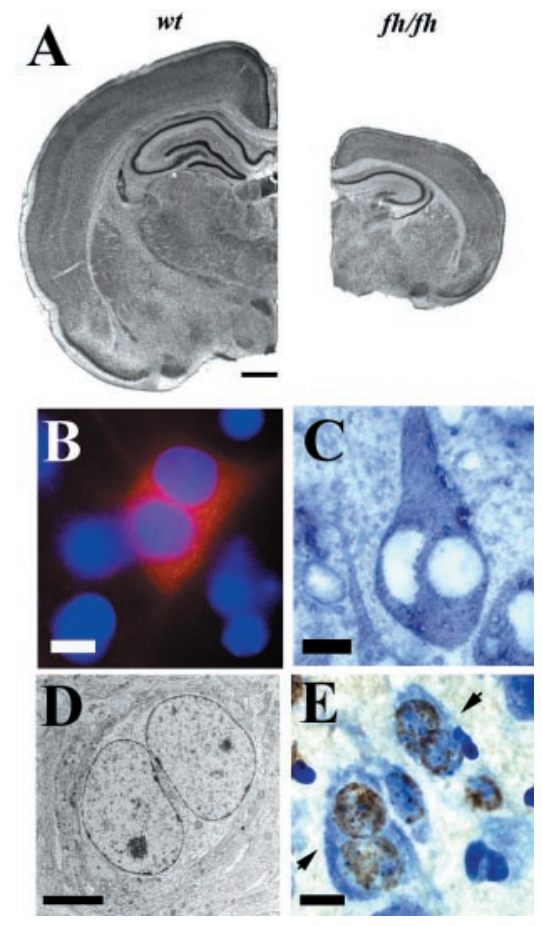

Figure 1. Reduced brain size and binucleate cells in $f h / f h$. $A$, Nisslstained coronal sections through P21 wt (left) and fh/fh (right) cortex. $B$, Binucleate GABA+ cell (red) containing two DAPI-labeled nuclei (blue) and a binucleate pyramidal cell $(C)$ labeled with an anti-rat brain pyramidal cell antibody (Swant, Bellinzola, Switzerland) in P14 neocortex. $D$, Electron micrograph showing a binucleate interneuron in stratum radiatum of hippocampus with no plasma membrane dividing the two nuclei. $E$, After an injection of BrdU at E15 and examination of neocortex at P12, many cells contain two nuclei, comparably labeled with BrdU (arrowheads). Scale bars: $A, 1000 \mu \mathrm{m} ; B-D, 5 \mu \mathrm{m} ; E, 10 \mu \mathrm{m}$.

University Faculty of Medicine, Kyoto, Japan), a polyclonal rabbit antiCitron (1:500) (Di Cunto et al., 2000), monoclonal mouse anti-RhoA (1:10) (Santa Cruz Biotechnology, Santa Cruz, CA), mouse-anti-TUJ1 (1:20,000) (Babco, Richmond, CA), and mouse anti-RAT401 (Nestin) (1:200) (Developmental Hybridoma Study Bank, Iowa City, IA). Secondary antibodies used were biotinylated goat anti-rabbit (Vector, Burlingame, CA), Alexa488-conjugated goat anti-rabbit (Molecular Probes, Eugene, OR), or Texas Red or Alexa594 goat anti-rabbit or goat anti-mouse (Molecular Probes). Nuclei were also stained with 4',6-diamidino-2-phenylindole dihydrochloride (DAPI) (Molecular Probes) (1:50,000). Electron microscopy procedures, Nissl staining, and immunohistochemistry for GABA, rat brain pyramidal cells, and bromodeoxyuridine (BrdU) were performed as described in previous studies (Roberts et al., 2000; Sarkisian et al., 2001). Epifluorescent images were obtained on a Nikon Eclipse E400 microscope using a Spot Digital camera, and confocal images were obtained using a Leica TCS SP2 Spectral Confocal Imaging System.

For immunocytochemistry of acutely dissociated cells, embryos were harvested at E14, and brains were dissected into cold HBSS (Invitrogen). Cerebral hemispheres from individual rats were isolated, and the ganglionic eminences were removed. The remaining neocortical $\mathrm{VZ}$ was placed into media containing $10 \%$ fetal bovine serum, $1 \%$ penicillin/ streptomycin, and 90\% S-MEM (Invitrogen). Cells were dissociated, plated onto protamine-coated coverslips, and allowed to plate at $37^{\circ} \mathrm{C}$, $5 \% \mathrm{CO}_{2}$ for $2 \mathrm{hr}$. Cells were fixed then with $4 \% \mathrm{PF}$, washed several times with PBS, and stained immunocytochemically as described above.

\section{RESULTS}

\section{Cytokinesis failure in the Flathead mutant}

In previous studies we have shown that the $f h / f h$ phenotype includes dramatically reduced brain size (Roberts et al., 2000) and the presence of many binucleate neurons (Mitchell et al., 2001; Sarkisian et al., 2001). Figure $1 A$ shows that at P21 the 
forebrain of $f h / f h$ mutants is approximately one-half the size of $w t$ rats, a difference that is present as early as P0 (Roberts et al., 2000). As shown in Figure $1 B-D$, the neocortex of $f h / f h$ mutants contains both binucleate nonpyramidal (Fig. $1 B$ ) and pyramidal neurons (Fig. $1 C$ ), and in electron micrographs the two nuclei are not separated by plasma membrane (Fig. $1 D$ ). Binucleate neurons are present throughout the CNS, including striatum, thalamus, hippocampus, midbrain, hindbrain, cerebellum, and spinal cord. To confirm that binucleate cells result from failed cytokinesis, we injected BrdU at E15 and examined $f h / f h$ neocortex at P12. We find nuclei within binucleate neurons that comparably label with BrdU (Fig. 1E, arrowheads), suggesting that both nuclei are generated at the same S-phase and that many neuronal progenitors in $f h / f h$ mutants fail to divide.

\section{The flathead gene is a null mutant allele of Citron-K}

To identify candidate genes that may contain the flathead mutation, we examined the region of human chromosome 12 between Nos-1 and TCF-1, the region syntenic to the region of rat chromosome 12 where we previously mapped the flathead mutation (Cogswell et al., 1998). The Citron gene, $\sim 2 \mathrm{Mb}$ telomeric from Nos1 and $2 \mathrm{Mb}$ centromeric from TCF-1, is the only gene within this region that has been specifically implicated in cytokinesis. To test the hypothesis that the Citron gene is mutated in flathead rat, we determined both whether the Citron gene in rat colocalizes with the flathead mutation and whether there is a mutation in Citron in $f h / f h$ mutants. We isolated and sequenced the entire cDNA sequence encoding Citron-K from both $f h / f h$ and $w t$ rats, and there is a deleted $\mathrm{G}-\mathrm{C}$ base pair in the kinase domain of fh/fh mutants (Fig. $2 A$ ). We also find the same base-pair deletion within clones isolated from exon 1 of $f h / f h$ genomic DNA (chromatograms not shown). In addition, we find that the kinase domain of Citron-K gene is present in three P1 clones in a P1 contig of rat chromosome 12 that contains D12Rat55, which maps to the flathead mutation. Together, these results suggest that the flathead mutation is a single base deletion in the first exon of the Citron-K gene.

The deleted base pair in exon 1 (Fig. $2 A$ ) would be expected to cause a shift in the reading frame resulting in a premature stop codon 10 codons downstream from the site of the mutation in exon 1 and 27 codons from the start ATG. The Citron gene has an unusual two-promoter structure in which transcription of the two primary gene products, Citron-K and Citron-N, are initiated from two separate promoters (Di Cunto et al., 2000). Citron-N transcripts are thought to be initiated from a promoter downstream from the kinase domain, and Citron-K transcripts are thought to be initiated from a promoter upstream from the kinase domain (Di Cunto et al., 2000). Citron-K transcript and protein are produced early in neural development, whereas Citron-N is produced predominantly in the postnatal brain (Furuyashiki et al., 1999; Zhang et al., 1999). On the basis of this mechanism of expression, the mutation in exon 1 in $f h / f h$ mutants would be expected to cause a specific elimination of Citron-K protein without affecting Citron-N protein. Consistent with this hypothesis, Citron-K protein is absent in E13 forebrain from mutants, during a time when only Citron-K is expressed (Fig. $2 B$ ). Immunocytochemical analysis further shows the lack of immunopositivity for Citron-K at the VZ surface of E14 neocortex in homozygous mutants (Fig. $2 C$ ). Similarly, Citron-K protein is absent in $\mathrm{P} 1 \mathrm{fh} / \mathrm{fh}$ cerebellum (Fig. $2 B$ ); however, Citron-N is expressed in both $w t$ and $f h / f h$ postnatal cerebellum and cortex (Fig. 2B). Because we do not have an antibody that can recognize the short
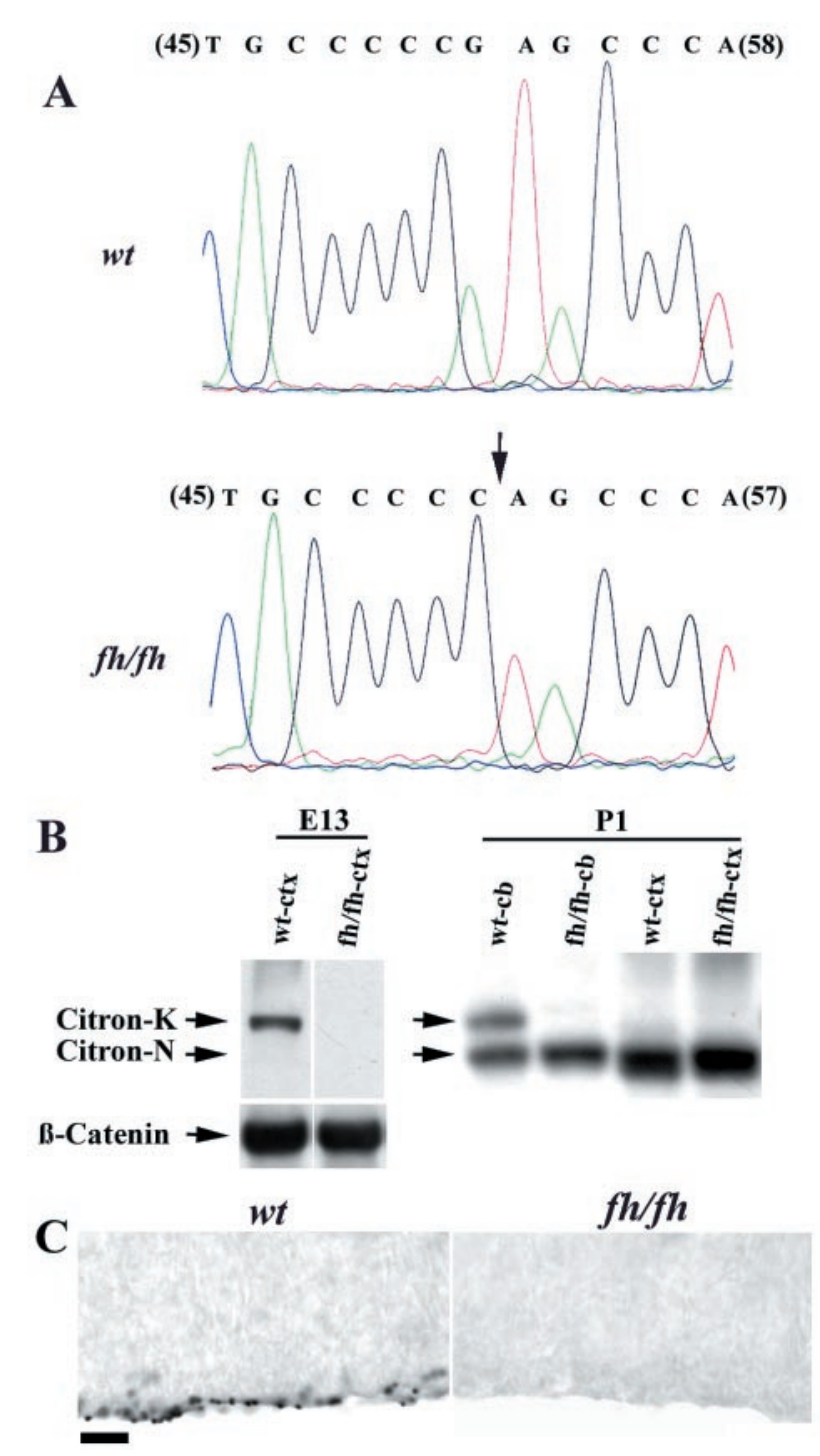

Figure 2. $f h$ is a null mutation in the Citron-K gene. $A$, Chromatograms showing cDNA sequence results within wt (top) and fh/fh (bottom) of the $\mathrm{N}$-terminal kinase domain of Citron-K. The arrow indicates a deleted G in $f h / f h$. Numbers in parentheses are base numbers relative to the Citron-K start ATG. $B$, Western blots show that Citron-K protein is absent in E13 $f h / f h$ cortex $(c t x)$ and P1 cerebellum $(c b)$. Citron-N levels in P1 $f h / f h$ and $w t$ are comparable in postnatal $\mathrm{ctx}$ and $\mathrm{cb} . \beta$-Catenin levels, in contrast, are not different. $C$, In E14 $w t$ forebrain (left), Citron-K expression is concentrated along the ventricular surface in the form of discrete punctate staining. This expression pattern is completely absent in $f h / f h(r i g h t)$. Scale bar, $10 \mu \mathrm{m}$.

peptide that could theoretically be produced in the flathead mutant, we cannot rule out the possibility that a short peptide is still produced in mutants. However, this small peptide (27 amino acids) should not be capable of having functional kinase activity because the catalytic domain would be completely missing (Madaule et al., 1998). Therefore, as predicted from the premature stop codon in exon 1 of the Citron gene, and confirmed with immunohistochemistry and Western blot analyses, the flathead mutation is a null mutant allele of the Citron-K gene.

Citron-K localizes to cleavage furrows of both symmetrically and asymmetrically dividing progenitors

Citron-K protein has previously been shown to localize to cytokinesis furrows in cell lines (Madaule et al., 1998). We performed 
immunocytochemistry experiments on cryosections and acutely dissociated cells from E13 and E14 neocortex to determine the expression of Citron-K in neuronal progenitors. At this time in neocortical development, neurons are primarily generated, and Citron-K and not Citron-N message (Di Cunto et al., 2000) and protein (Fig. 2B) are expressed. Citron-K protein in E14 wt is concentrated along the entire VZ surface (Fig. 2C, left panel) and is also found throughout the external granular layer of early postnatal cerebellum (data not shown). Homozygous mutants completely lack staining in proliferative regions (Fig. $2 C$, right panel) further indicating that Citron-K and not Citron-N protein is expressed in neuronal progenitors. Citron- $\mathrm{K}$ protein is present at cytokinesis furrows between dividing cells at the VZ surface, and Citron-K immunoreactivity perfectly outlines furrows that appear to be in different stages of cytokinesis. Furrows typically

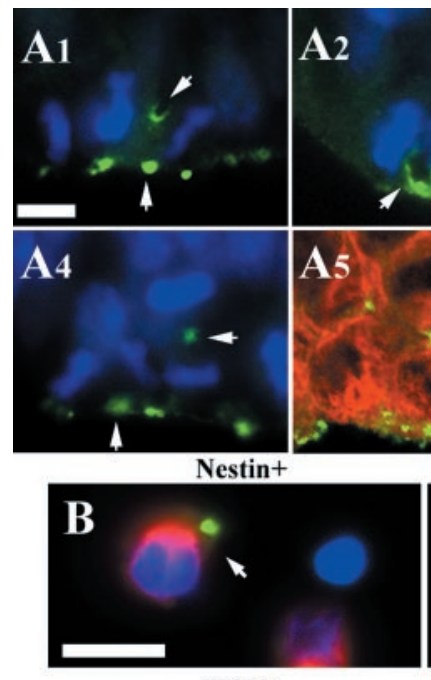

TUJ1+
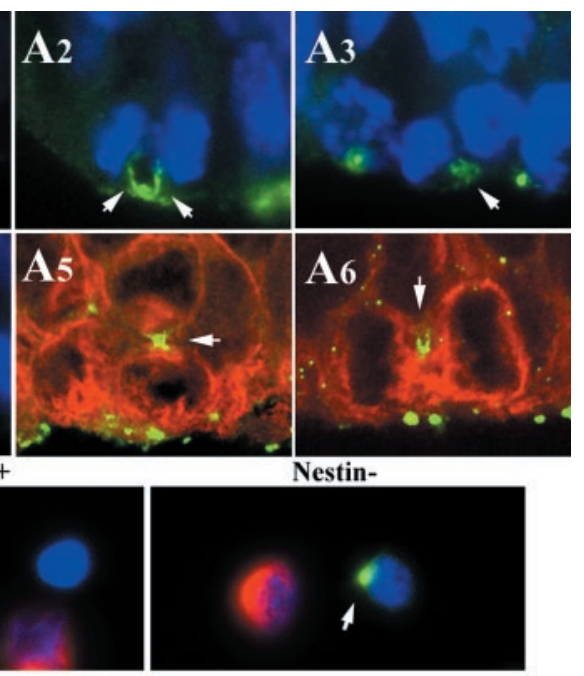

TUJ1-
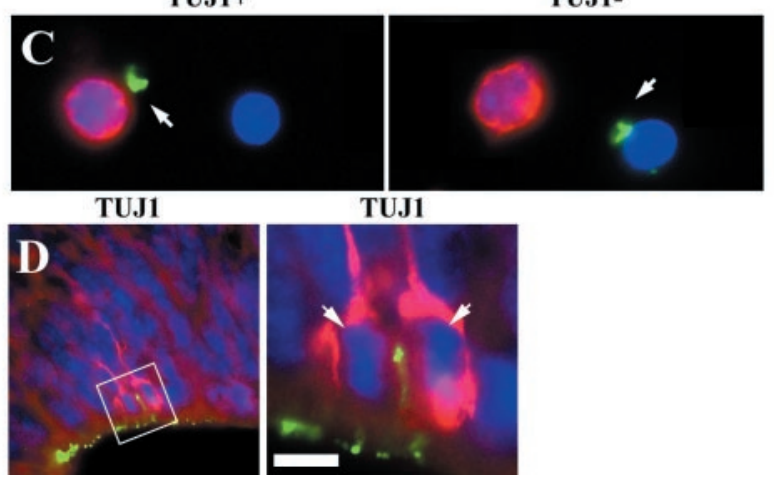

Figure 3. Citron-K expression at cytokinesis furrows in the cortical ventricular surface at E13 $(A)$ and E14 $(B-D) . A 1$, Citron-K is expressed at the basal side of radially dividing cells in a U-shaped pattern (top arrowhead) that seems to pull toward Citron-K at the VZ surface (bottom arrowhead). A2, Citron-K forms membranous staining patterns (arrowheads) on either side of cleavage furrows in late telophase. A3, Citron-K at a cleavage furrow (arrowhead) of late telophase when nuclei in each daughter have reassembled. A4, Horizontally dividing cell in the VZ with Citron-K at the furrow (arrowheads). A5, A6, Confocal images of double immunoreactivity for Nestin (red) and Citron-K (green) in horizontally $(A 5)$ and vertically $(A 6)$ dividing cells showing Citron-K at the furrows (arrows). B, $C$, Expression of Citron-K on the surface of Nestin $+(B$, left $)$ and Nestin $-(B$, right $)$ cells and TUJ1+ $(C$, left $)$ and TUJ1 $-(C$, right $)$ cells from E14 neocortical VZ. D, Low magnification of TUJ1 and Citron-K coimmunoreactivity $(D$, left $)$. The boxed inset $(D$, left $)$ shows a higher magnification of Citron-K expression between a dividing pair of TUJ1+ cells $(D$, right $)$. Scale bars: $A, D, 5 \mu \mathrm{m} ; B, C, 10 \mu \mathrm{m}$.

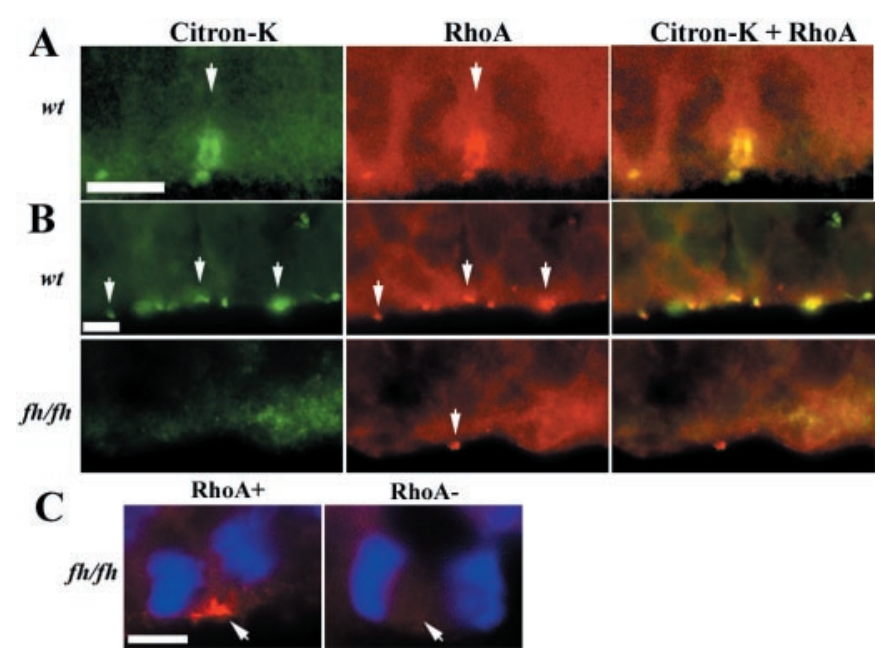

Figure 4. RhoA colocalizes with Citron-K at the VZ surface and is normally and abnormally localized in $\mathrm{fh} / \mathrm{fh}$. $\mathrm{A}$, Confocal image of Citron-K (left, arrowhead) and RhoA (middle, arrowhead) at a cleavage furrow in E13 wt forebrain. Citron-K and RhoA colocalize at the furrow (right, yellow). B, Citron-K (left, arrowheads) and RhoA (middle, arrowheads) are colocalized (right, yellow) along the VZ surface in E13 wt (top panels) compared with fh/fh (bottom panels), which shows not only an absence of Citron-K but dramatically fewer RhoA puncta (arrowhead in bottom panel ). C, Examples of dividing nuclear profiles in E13 fh/fh with cytokinesis furrows with (left, arrowhead) and without RhoA (right, arrowhead). Scale bars, $5 \mu \mathrm{m}$.

form at both the basal and apical poles of dividing cells, then the basal aspect of the furrow appears to pull asymmetrically down toward the apical, VZ surface (Fig. 3A1-A3). We also find some Citron-K-labeled furrows that are horizontally oriented to the VZ surface (Fig. 3A4,A5). In horizontally dividing cells, unlike radially dividing cells, furrows appear to form on lateral sides of the cell and pull symmetrically toward the midbody (Fig. 3A5). This expression pattern indicates that Citron-K is a useful marker for cytokinesis furrows in CNS progenitors and may be used to specifically identify sibling pairs of recently generated daughter cells. Double immunocytochemistry in acutely dissociated E14 VZ cells show Citron puncta associated with both Nestin (Fig. $3 B$ ) and TUJ1 (Fig. 3C) positive cells. Similarly, in tissue sections, Citron-K is present at cytokinesis furrows occurring between pairs of intensely Nestin + (Fig. 3A5,A6) or TUJ1+ (Fig. 3D) cells. Because Citron-K is present at the cytokinesis furrows of both radially and horizontally dividing progenitors and is expressed by Nestin + and TUJ1+ cells, Citron-K appears to be involved in cytokinesis of both symmetrically and asymmetrically dividing precursors.

\section{Citron-K colocalizes with RhoA at the VZ surface}

Activated RhoA directly binds to Citron-K and increases kinase activity by eightfold (Di Cunto et al., 1998). In HeLa cells, RhoA and Citron-K colocalize at cytokinesis furrows, mutants of Citron-K that lack the RhoA binding domain disrupt cytokinesis, and activated RhoA is necessary to localize Citron-K/RhoA to cleavage furrows (Madaule et al., 1998; Eda et al., 2001). Similarly, in developing neocortex we found that RhoA colocalizes with Citron-K at cleavage furrows and suspected midbodies at the VZ surface(Fig. $4 A$ ). In contrast to the pattern of RhoA at the VZ surface in $w t$ neocortex (Fig. 4B, top panels), RhoA at the VZ surface of $f h / f h$ mutant embryos is sparse (Fig. $4 B$, bottom panels), and many cytokinesis furrows and midbodies in homozygous 
mutants lack RhoA (Fig. 4C). This indicates that either RhoA expression levels or RhoA distribution is altered at cytokinesis furrows in $f h / f h$ mutants. Intense RhoA immunoreactivity, however, could still be identified in a few radial (Fig. 4C) and horizontally (data not shown) oriented divisions in mutants. These results indicate that RhoA is colocalized with Citron-K at the VZ surface and that Citron-K may be necessary for normal RhoA distribution in some dividing progenitors.

\section{DISCUSSION}

\section{Citron-K knock-out mice and fh/fh mutant rats}

The Citron-K mutation in mice results in a phenotype that is nearly identical to the $f h / f h$ rat (Di Cunto et al., 2000), and this further confirms that the mutation in Citron-K that we have identified is the $f h / f h$ mutation. Remarkably this knock-out mouse was independently generated by disrupting exon 2 with homologous recombination merely 88 base pairs from the site of the $f h$ mutation. In both the mutant rat and mouse, Citron-N protein is produced, whereas Citron-K is absent. The major phenotypic differences between Citron-K $-/-$ mice and $f h / f h$ rats include severely disrupted retinal development (Roberts et al., 2000) and a more severely disrupted internal granular layer of cerebellum in $f h / f h$ rats. These relatively minor differences may reflect species differences in the requirement for Citron-K. In both mutants, there is massive apoptosis in proliferative regions during mid-embryonic and early postnatal periods (Di Cunto et al., 2000; Roberts et al., 2000; Mitchell et al., 2001; Sarkisian et al., 2001). Cytokinesis failure in both mutants precedes apoptosis, suggesting that the increased apoptosis is caused by failed cytokinesis (Di Cunto et al., 2000; Mitchell et al., 2001; Sarkisian et al., 2001). In the present study, the localization of Citron-K at cytokinesis furrows is further evidence that the primary defect in the $f h / f h$ mutant is in cytokinesis. It is not presently clear why $4 \mathrm{~N}$ cells should have a higher incidence of death, but it may indicate a mechanism to eliminate failed cytokinesis in normal development.

\section{Polarization of Citron-K and cytokinesis}

Citron-K protein is localized to the ventricular surface (Fig. $3 A$ ) at cytokinesis furrows and to smaller puncta at the very lumenal surface. We hypothesize based on transitional cytokinesis profiles, and the pattern of midbodies described in electron microscopic analyses by Hinds and Ruffett (1971), that the small puncta are midbodies that remain attached to the VZ surface after newly generated daughters migrate away from the VZ surface. Citron-K is localized to the VZ surface by as yet unidentified complexes; however, the protein structure of Citron-K may indicate possible mechanisms of polarization (Madaule et al., 2000). The $\mathrm{C}$-terminal end of Citron has a consensus PDZ binding domain (QSSV), and many of the proteins at the ventricular surface, including adherens junctions proteins, contain PDZ proteins (Chenn et al., 1998). In future studies, determining how Citron-K is maintained at the $\mathrm{VZ}$ surface may lead to insights into how mitoses in the VZ are localized to the VZ surface.

\section{Additional mechanisms for the molecular control of cytokinesis in the VZ}

Recently, single radial glial cells in the VZ have been shown to give rise to both neurons and radial glial progenitors (Miyata et al., 2001; Noctor et al., 2001). We found Citron-K in Nestin + and TUJ1+ cells, and therefore Citron-K may be involved in cleav- ages within radial glial cells that eventually give rise to neurons. It is apparent that because many $f h / f h$ neurons, including many pyramidal and nonpyramidal neurons in neocortex, have only one nucleus (Sarkisian et al., 2001), Citron-K is not essential to all cytokineses. In addition, Citron-K is not expressed in neocortex during the period of greatest gliogenesis (Fig. 2B), suggesting that mechanisms of cytokinesis change through development and are different for different neural progenitors (Lu et al., 2000). We found that RhoA is colocalized with Citron-K at cytokinesis furrows in $w t$, and that in $f h / f h$ mutants, RhoA remains localized to some but not all cytokinesis furrows. These data suggest that RhoA may be localized by other proteins besides Citron-K, and perhaps these can replace Citron-K function in some dividing precursors. Because fate-determining signals are distributed to daughters during cell division, the apparent heterogeneity in cytokinesis mechanisms in the CNS may reflect different mechanistic requirements for generating different cell types.

\section{REFERENCES}

Chang F, Nurse P (1996) How fission yeast fission in the middle. Cell 84:191-194.

Chenn A, Zhang A, Chang BT, McConnell SK (1998) Intrinsic polarity of mammalian neuroepithelial cells. Mol Cell Neurosci 11:183-193.

Cogswell CA, Sarkisian MR, Leung V, Patel R, D'Mello SR, LoT urco JJ (1998) A gene essential to brain growth and development maps to the distal arm of rat chromosome 12. Neurosci Lett 251:1-4.

Di Cunto F, Calautti E, Hsiao J, Ong L, Topley G, Turco E, Paolo Dotto $\mathrm{G}$ (1998) Citron rho-interacting kinase, a novel tissue-specific ser/thr kinase encompassing the rho-rac binding protein citron. J Biol Chem 273:29706-29711.

Di Cunto F, Imarisio S, Hirsch E, Broccoli V, Bulfone A, Migheli A, Atzori C, Turco E, Triolo R, Paolo Dotto G, Silengo L, Altruda F (2000) Defective neurogenesis in citron kinase knockout mice by altered cytokinesis and massive apoptosis. Neuron 28:115-127.

Drees BL, Sundin B, Brazeau E (2001) A protein interaction map for cell polarity development. J Cell Biol 154:549-571.

Eda M, Yonemura S, Kato T, Watanabe N, Ishizaki T, Madaule P, Narumiya S (2001) Rho-dependent transfer of citron-kinase to the cleavage furrow of dividing cells. J Cell Sci 114:3273-3284.

Feng Y, Walsh CA (2001). Protein-protein interactions, cytoskeletal regulation and neuronal migration. Nat Rev Neurosci 2:408-416.

Field C, Li R, Oegema K (1999) Cytokinesis in eukaryotes: a mechanistic comparison. Curr Opin Cell Biol 11:68-80.

Furuyashiki T, Fujisawa K, Fujita A, Madaule P, Uchino S, Mishina M, Bito H, Narumiya S (1999) Citron, a rho-target, interacts with PSD95/SAP-90 at glutamatergic synapses in the thalamus. J Neurosci 19:109-118.

Hinds JW, Ruffett TL (1971) Cell proliferation in the neural tube: an electron microscopic and Golgi analysis in the mouse cerebral vesicle. $\mathrm{Z}$ Zellforsch 115:226-264.

Lu B, Jan L, Jan YN (2000) Control of cell divisions in the nervous system: symmetry and asymmetry. Annu Rev Neurosci 23:531-556.

Madaule P, Eda M, Watanabe N, Fujisawa K, Matsuoka T, Bito H, Ishizaki T, Narumiya S (1998) Role of citron kinase as a target of the small GTPase rho in cytokinesis. Nature 394:491-494.

Madaule P, Furuyashiki T, Eda M, Bito H, Ishizaki T, Narumiya S (2000) Citron, a rho target that affects contractility during cytokinesis. Microsc Res Tech 49:123-126.

Mitchell BD, Gibbons B, Allen LR, Stella J, D’Mello SR (2001) Aberrant apoptosis in the neurological mutant Flathead is associated with defective cytokinesis of neural progenitor cells. Dev Brain Res 130:53-63.

Miyata T, Kawaguchi A, Okano H, Ogawa M (2001) Asymmetric inheritance of radial glial fibers by cortical neurons. Neuron 31:727-741.

Noctor SC, Flint AC, Weissman TA, Dammerman RS, Kriegstein AR (2001) Neurons derived from radial glial cells establish radial units in neocortex. Nature 409:714-720.

Roberts MR, Bittman K, Li W, French R, Mitchell B, LoTurco JJ, D'Mello SR (2000) The flathead mutation causes CNS-specific developmental abnormalities and apoptosis. J Neurosci 20:2295-2306.

Sarkisian MR, Frenkel M, Li WW, Oborski JA, LoTurco JJ (2001) Altered interneuron development in the cerebral cortex of the flathead mutant. Cereb Cortex 11:734-743.

Zhang W, Vazquez L, Apperson M, Kennedy MB (1999) Citron binds to PSD-95 at glutamatergic synapses on inhibitory neurons in the hippocampus. J Neurosci 19:96-108. 